\title{
Cooling in HEP Vertex and Tracking Detectors
}

\section{Hans Postema ${ }^{1}$}

CERN

1211 Geneva 23, Switzerland

E-mail: hans.postema@cern.ch

\section{Bart Verlaat}

Nikhef (Currently based at CERN)

Science Park 105, 1098 XG, Amsterdam, The Netherlands

E-mail: bverlaat@nikhef.nl

A large variety of cooling systems for the detectors mentioned above, currently exist. Each of them have particular advantages and challenges. We compare the different systems of the LHC experiments and focus on $\mathrm{CO}_{2}$ cooling which is now considered a prime candidate for future cooling systems including upgrades for LHC detectors. Starting with a description of the $\mathrm{CO}_{2}$ systems that are currently operational in detectors we continue with an overview of the many prototypes and test systems that are currently available or under construction. Finally we summarize the important ingredients that are needed in order to achieve successful and reliable operation of a cooling system.

VERTEX 2011(20 th workshop) - VERTEX 2011

Rust, Austria

June 19-24, 2011

\footnotetext{
1 Speaker
} 


\section{Introduction}

Before discussing a cooling system, it is good to contemplate on the question: "How does a Tracking detector look, from a cooling system point of view?" Using the CMS tracker as an example, one sees that we have a highly distributed heat load of about $35 \mathrm{~kW}$, serviced by more than 4000 meters of cooling tube with diameters between 2 and $3 \mathrm{~mm}$. Operation is at $-20{ }^{\circ} \mathrm{C}$ or lower and the detector typically needs supply and return lines with lengths of 50 meters. Other detectors vary in size but the principal issues remain the same. The goals to achieve are reliable cooling at temperatures between $-40{ }^{\circ} \mathrm{C}$ and $+20^{\circ} \mathrm{C}$ and at the same time reducing the amount of material inside the active volume to the minimum. Several engineering issues complicate the task. The choice of coolants is limited by the radiation in the detector. The coolant shall be as radiation hard as possible and the products of radiation damage should not be corrosive in order to avoid damage to the tubing. Radiation shall also not create polymerisation, which could lead to a reduced heat transfer or blockage of thin tubes. Radiation as well as magnetic field, limit the choice of components. One must also keep in mind that the system is often inaccessible during long periods of time. Depending on the location, the time between accesses can be years. Reliability is served by minimising the number of components, especially the number of active components. Active components shall be located as much as possible, in accessible places. A variety of systems is currently in operation at LHC.

\section{Operational cooling systems in LHC}

The 4 main LHC detectors Alice, Atlas, CMS and LHCb have all different methods to cool their inner tracking detectors. Below an overview of the different cooling systems used is given.

\subsection{ALICE-SPD}

The ALICE-SPD uses a two-phase system using $\mathrm{C}_{4} \mathrm{~F}_{10}$ as the working fluid [1]. The normal working temperature is $+15 \mathrm{C}$, resulting in an operation pressure of 1.9 bar absolute. This low pressure does not allow for much pressure drop in the return line, which as a result needs a larger diameter. This can be problematic in places where the space for detector services is limited, which is often the case. The system uses chilled water as the primary cold source, which uses standard industrial technology.

\subsection{ATLAS - SCT}

This detector uses a two-phase system using $\mathrm{C}_{3} \mathrm{~F}_{8}$ as the working fluid [2]. The normal operating temperature is $-25 \mathrm{C}$, resulting in an operating pressure of 1.7 bar absolute, also a low pressure that leaves little margin for pressure losses along the return line, thus requiring a larger pipe diameter. This system works with supply and return lines at room temperature, which is a distinct advantage but requires the presence of carefully controlled heaters. The system uses the $\mathrm{C}_{3} \mathrm{~F}_{8}$ in a vapour compression cycle. Due to the radiation, one is not allowed to have oil in the 
refrigerant, which is the standard in the refrigeration industry. This complicates the choice for suitable compressors.

\subsection{CMS Tracker}

This detector uses a single phase system with $\mathrm{C}_{6} \mathrm{~F}_{14}$ as the working fluid [3]. The higher mass flow in a single phase system requires larger supply and return lines. The operating temperature is $-20^{\circ} \mathrm{C}$ and the operating pressure is 6 to 9 bar absolute. The simple design and the use of an industrial standard R507a chiller as primary cold source are important advantages.

\subsection{LHCb VELO}

This detector uses a 2-phase cooling system using $\mathrm{CO}_{2}$ as the working fluid [4]. The operating temperature is $-30^{\circ} \mathrm{C}$, resulting in an operating pressure of 14 bar absolute. The system is designed for maximum simplicity and robustness and uses industrial standard R507a chiller as primary cold source.

\section{Why is evaporative $\mathrm{CO}_{2}$ cooling good for $\mathrm{HEP}$ detectors?}

$\mathrm{CO}_{2}$ has several physical properties which all combine together, lead to an excellent performance when used in small cooling tubes such as one needs in Vertex detectors. The gain of radiation length of the cooling system hardware when compared to other common fluids used in particle detector cooling is an order of magnitude in favour of $\mathrm{CO}_{2}$ [5]. The benefits of $\mathrm{CO}_{2}$ are the low viscosity, the large latent heat and the high pressure. The latter looks like a disadvantage but is actually an advantage as pressure drop becomes less significant. A pressure drop in a pipe results in a decreasing pressure along the pipe and consequently a decreasing boiling temperature. The ratio of the temperature drop as a function of pressure drop is fluid dependant. The comparison of latent heat, liquid viscosity and dT/dP ratio are plot in figure 1 .
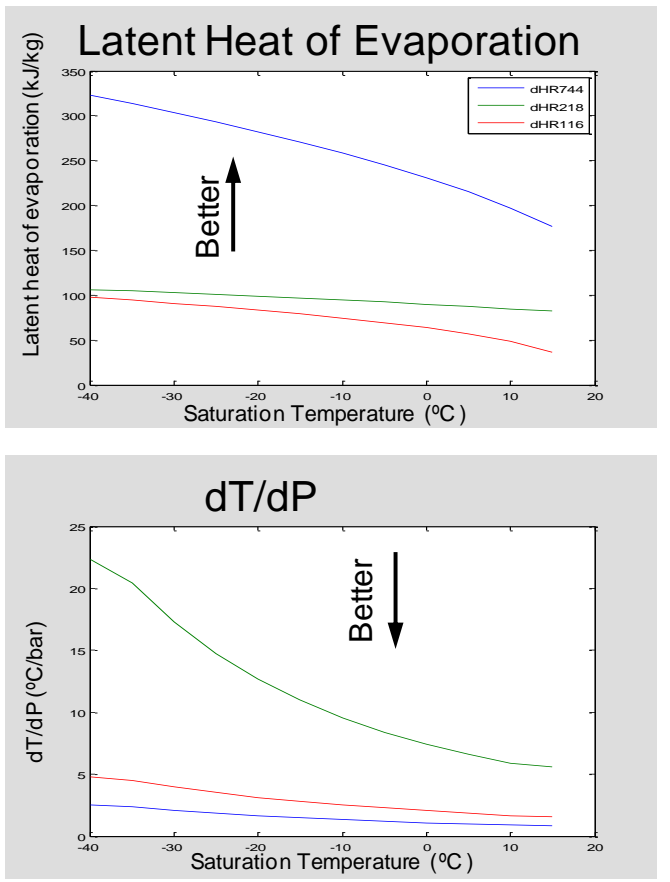

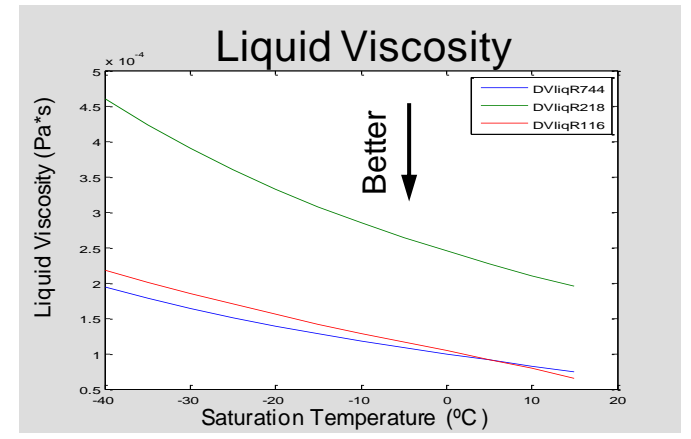

Figure 1: Property comparison between $\mathrm{CO}_{2}$ (Blue, R744), $\mathrm{C}_{3} \mathrm{~F}_{8}$ (Green, R218) and $C_{2} F_{6}(R e d, R 119)$ 
The temperature in the detector and hence the cooling tube must in general be as uniform as possible. Taking the above property comparison in mind, $\mathrm{CO}_{2}$ is clearly superior compared to fluorocarbons. The high latent heat requires a small mass flow resulting in low pressure drops. The low viscosity results as well in a lower pressure drop, while for a similar temperature uniformity a higher pressure drop is allowed since the temperature/pressure gradient ratio is much higher. All together it leads to the ability of using very small tubes for $\mathrm{CO}_{2}$ evaporative systems.

\section{4. $\mathrm{CO}_{2}$ and safety}

Frequently, concern is expressed because of the high pressures involved when using $\mathrm{CO}_{2}$ systems. Safety regulations such as the Pressure Equipment Directive (PED) [6] however, correctly address the problem as a function of stored energy, which is the pressure multiplied by the volume. Apart from the accumulators, which can be safety critical devices in the higher classes of the PED, all other components are generally below class one and do not require special certification procedures, although good workmanship remains a requirement. As stated the safety class is determined by the internal stored energy (See figure 2). The pressures in $\mathrm{CO}_{2}$ systems have an order of magnitude higher design pressures, but the volume is, due to the small piping, an order of magnitude lower. The stored energy and hence the safety impact is therefore not much different from that of low pressure systems with higher volumes.

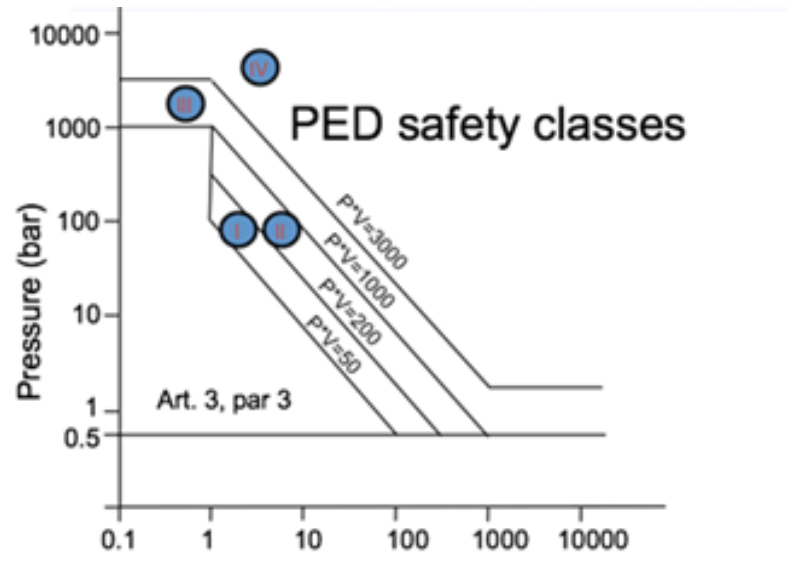

Figure 2: PED safety classes as a function of stored energy. Tubes below $32 \mathrm{~mm}$ are Article 3.3.
Recently the existing copper tubing in CMS has been certified for the use with $\mathrm{CO}_{2}$. A representative setup using identical tubing and identical brazing techniques was first carefully tested in order to identify the elastic limit and was then pressurized up to rupture. For the $14 \times 12 \mathrm{~mm}$ tube we found an elastic limit around 120 bar and a rupture at more than 240 bar. Since large systems in underground caverns are not expected to arrive at a temperature higher than $25^{\circ} \mathrm{C}$, we remain in the subcritical region and

the pressure will thus remain below 65 bar, since the system is only partially filled with liquid. A safely valve setting at 70 bar can thus be considered sufficient and an acceptance test at $1.25 \times 70=87.5$ bar is acceptable to CERN safety. Since this is not an industrial installation the European PED does not have to be followed.

\section{5. $\mathrm{CO}_{2}$ systems in HEP}

Actually, two $\mathrm{CO}_{2}$ cooling systems have been developed for HEP detectors and are in operation. For the first system, the AMS-TTCS (Tracker Thermal Control System) [7], the 
development started already back in 1999. The experiment is installed on the International Space Station and is operating in space since 20 May 2011 (figure 3). The cooling power is 150 Watt and the operating temperature is adjustable between $+15^{\circ} \mathrm{C}$ to $-20^{\circ} \mathrm{C}$.

The development of the second system, the LHCb-VTCS (Velo Thermal Control System) [6] started somewhat later but this system became operational before AMS. It has been continuously operating at $-30^{\circ} \mathrm{C}$ since 2008 . The cooling power is 1500 Watt (2 parallel systems of $750 \mathrm{~W})$ and the operating temperature can be adjusted from $+8^{\circ} \mathrm{C}$ to $-30^{\circ} \mathrm{C}$. Both systems are based on the 2PACL principle developed at NIKHEF.

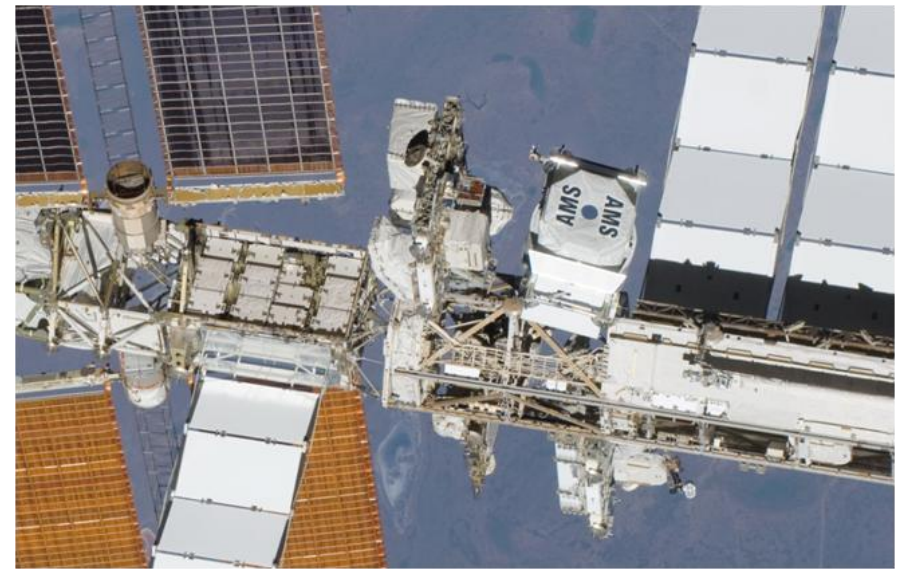

Figure 3: The first $\mathrm{CO}_{2}$ system in Particle Physics and the 1st $\mathrm{CO}_{2}$ system in Space: The AMS-Tracker Thermal Control Svstem

\section{Ongoing projects}

Currently five detectors envisage an upgrade employing $\mathrm{CO}_{2}$ cooling. They are: Atlas IBL, CMS-pixel replacement, Atlas and CMS silicon detector upgrade and the Belle-2 detector at KEK. At the moment there are at least 11 closed loop $\mathrm{CO}_{2}$ cooling platforms for testing either operational or under construction. These systems are: CERN B187 test setup, CERN-CV ATLAS plant at SR1, the Fermilab CMS FPIX cooling plant, the IPNL Lyon CMS test setup, the NIKHEF 2PACL system and RWTH Aachen CMS cooling test setup as well as the CERN Cryolab 2PACL system and the CERN-DT / Nikhef $1 \mathrm{~kW}$ and 100W system which are shown in figure 4. The systems at CERN and NIKHEF are open to external customers.
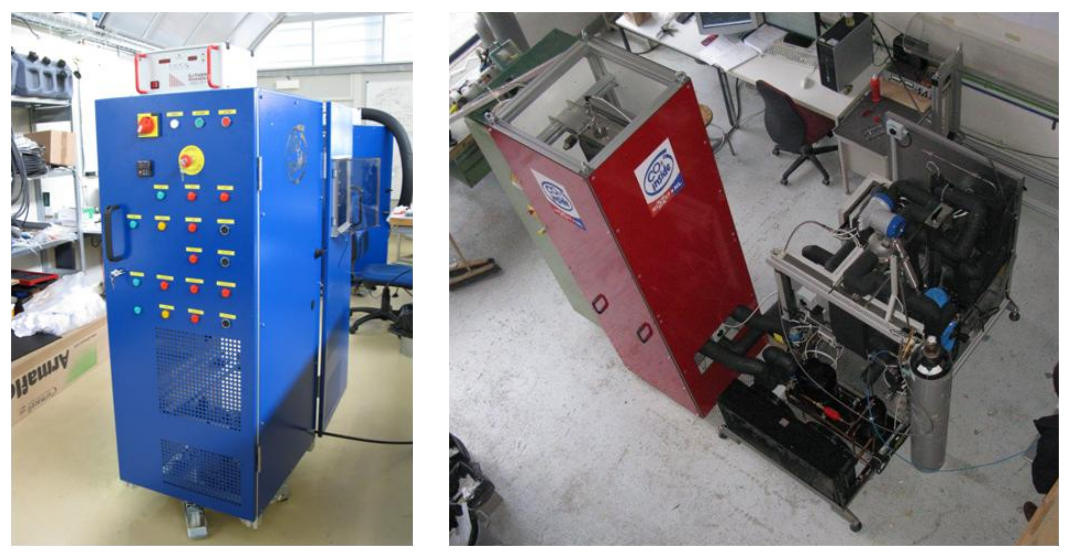

Figure 4: $\mathrm{CO}_{2}$ Test systems at CERN. Left is the TRACI system (100W), the middle the Cryolab plant CORA(2kW) and right the MARCO svstem $(1 \mathrm{~kW})$ which is under construction.

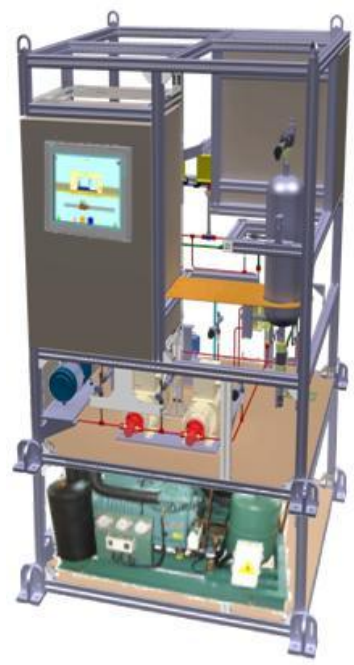




\section{7. $\mathrm{CO}_{2}$ Collaboration Partners}

There are a large number of groups currently working on the development of $\mathrm{CO}_{2}$ cooling. The collaboration is based upon a free exchange of information, sharing results as well as good and bad experiences. In alphabetic order: CERN-CMS, CERN-Cryolab, CERN-CV-Atlas, CERN-DT, EPFL Lausanne, Fermilab-CMS, HEPHY Vienna - Belle II, IPNL-Lyon - CMS, KEK Japan - Belle II, NIKHEF, NLR Amsterdam - AMS, Karlsruhe University, MPI Muenchen - Belle II, PSI Villingen - CMS, RWTH Aachen - CMS, SLAC - Atlas.

\section{Cooling Success}

A successful and reliable cooling system is not only depending on the selection of the right working fluid. Choosing a concept that uses the maximum amount of industrial experience and industrial technology assures maximum reliability. The system shall also be designed, keeping the very limited access in mind. Using components of a high quality will increase the initial cost somewhat but will generate the lowest overall costs in the long run. Fluids can be expensive, maintenance and refurbishments costs can be very substantial. Currently used coolants, such as $\mathrm{C}_{3} \mathrm{~F}_{8}$ and $\mathrm{C}_{6} \mathrm{~F}_{14}$ have high global warming potentials and might be discontinued in the future due to environmental concerns. In very large systems, they are also an important cost item, $\mathrm{C}_{6} \mathrm{~F}_{14}$ costs up to 100 SFR per litre where $\mathrm{CO}_{2}$ costs only 1 SFR per litre.

\section{Conclusions}

$\mathrm{CO}_{2}$ cooling can be the optimal solution for cooling VERTEX and Tracking detectors. A successful and reliable cooling system requires a well chosen concept, a simple and excellent design and high quality components and manufacturing.

\section{References}

[1] Pepato, A. et al, The mechanics and cooling system of the ALICE silicon pixel detector, Nuclear Instruments and Methods, Volume 565, Issue 1, 2006, Pages 6-12

[2] Attree D. et al, The evaporative cooling system for the ATLAS inner detector, 2008 Jinst 3 P07003.

[3] P.Tropea, Technical ADDENDUM to the Technical Specification for the cooling plant for the CMS Tracker and Preshower detectors, IT3230/TS/CMS, CERN EDMS 480997v4

[4] Verlaat B. et al, $\mathrm{CO}_{2}$ cooling for the LHCb-VELO experiment AT CERN", 8th IIF/IIR Gustav Lorentzen Conference on Natural Working Fluids (GL-2008), Copenhagen, Denmark, CDP 16-T308

[5] Verlaat, B. et al, $\mathrm{CO}_{2}$ Cooling Developments for HEP Detectors, 18th International Workshop on Vertex detectors, (VERTEX-2009), The Netherlands, PoS 031

[6] European Commission, Pressure Equipment Directive (97/23/EC), http://ec.europa.eu/enterprise/sectors/pressure-and-gas/documents/ped/

[7]Van Es, J. et al, AMS02 Tracker Thermal Control System overview and spin-off for future spacecraft cooling system developments, 60th International Astronautical Congress, Daejeon, South Korea, IAC-09.C2.7.1,2009 